\title{
Cardioplegia and Diazoxide Modulate STAT3 Activation and DNA Binding
}

\section{Citation}

Hsieh, Yng-Ju, Hidetaka Wakiyama, Sidney Levitsky, and James D. McCully. 2007. "Cardioplegia and Diazoxide Modulate STAT3 Activation and DNA Binding." The Annals of Thoracic Surgery 84 (4) (October): 1272-1278. doi:10.1016/j.athoracsur.2007.05.014.

\section{Published Version}

doi:10.1016/j.athoracsur.2007.05.014

\section{Permanent link}

http://nrs.harvard.edu/urn-3:HUL.InstRepos:27352041

\section{Terms of Use}

This article was downloaded from Harvard University's DASH repository, and is made available under the terms and conditions applicable to Other Posted Material, as set forth at http:// nrs.harvard.edu/urn-3:HUL.InstRepos:dash.current.terms-of-use\#LAA

\section{Share Your Story}

The Harvard community has made this article openly available.

Please share how this access benefits you. Submit a story.

Accessibility 


\title{
Cardioplegia and Diazoxide Modulate STAT3 Activation and DNA Binding
}

\author{
Yng-Ju Hsieh, PhD, Hidetaka Wakiyama, MD, Sidney Levitsky, MD, and James D. McCully, \\ PhD \\ Division of Cardiothoracic Surgery, Beth Israel Deaconess Medical Center, Harvard Medical \\ School, Harvard Institutes of Medicine, Boston, Massachusetts
}

\section{Abstract}

Background-Previously, we have shown that magnesium supplemented potassium (DSA) cardioplegia and DSA containing diazoxide (DSA+DZX) significantly decrease apoptosis after ischemia. The mechanism for this enhanced cardioprotection was unknown, but we believed that alterations in signal transducers and activators of transcription (STATs) may play a role. To investigate this hypothesis, we examined the effects of DSA and DSA+DZX cardioplegia on STAT1/3 phosphorylation and DNA binding in the in situ blood perfused pig heart model.

Methods-Pigs ( 32 to $42 \mathrm{~kg}$ ) undergoing total cardiopulmonary bypass underwent left anterior descending coronary artery occlusion for 30 minutes. The aorta was crossclamped and DSA ( $\mathrm{n}=$ 6) or DSA+DZX $(n=6)$ cardioplegia was administered, followed by 30 minutes of global ischemia and 120 minutes of reperfusion. Control hearts $(n=3)$ received cardiopulmonary bypass and sham reperfusion only. Tissue samples from regional and global ischemia zones were harvested and used for Western blot and electrophoretic mobility shift assay.

Results-Regional and global ischemia significantly increase proapoptotic STAT1 tyrosine phoshorylation. This increase is significantly greater in the regional as compared with the global ischemia zone. Tyrosine phosphorylation of antiapoptotic STAT3 is increased in the global ischemic zone but is significantly decreased in the regional ischemic zone and is associated with increased apoptosis. The DSA+DZX significantly increases tyrosine phosphorylation of antiapoptotic STAT3 and DNA binding in the regional ischemia zone and significantly decreases apoptosis.

Conclusions-The addition of diazoxide to DSA cardioplegia significantly decreases apoptosis by significantly increasing tyrosine phosphorylation of STAT3 and its DNA binding and represents an additional modality for enhancing myocardial protection.

Previous investigations have shown that the signal transducers and activators of transcription (STAT) signaling pathway plays an important role in the modulation of apoptosis after ischemia and reperfusion [1-5]. While the mechanism remains to be fully elucidated, present data suggest that STAT proteins normally present as latent cytoplasmic monomeric transcription factors are rapidly activated by phosphorylation at either serine or tyrosine residues $[6,7]$.

Cellular and in vitro studies have shown that activation of STAT1 by tyrosine phosphorylation and its DNA binding is associated with proapoptotic responses while the activation of STAT3 by tyrosine phosphorylation and its DNA binding is associated with 
antiapoptotic responses [1-5]. Whether these pathways are involved in the modulation of apoptosis in a clinically relevant blood perfused heart model of ischemia and reperfusion was unknown.

In previous studies, we have shown that magnesium supplemented potassium (DSA) cardioplegia significantly decreases apoptosis after ischemia $[8,9]$. We have also shown that the mitochondrial adenosine triphosphate (ATP)-sensitive potassium (mitoK $\mathrm{ATP}_{\mathrm{A}}$ ) channels play an important role in the cardioprotection afforded by DSA cardioplegia and that the early or enhanced opening of the mitoK $\mathrm{K}_{\mathrm{ATP}}$ channels with the addition of diazoxide to DSA cardioplegia (DSA+DZX) significantly decreases apoptosis as compared with DSA cardioplegia alone $[8,9]$. In the present study, we investigate the effects of ischemia and reperfusion and DSA and DSA+DZX cardioplegia on STAT1 and STAT 3 phosphorylation and DNA binding by extending our previous studies in the in situ blood-perfused pig heart model of acute myocardial infarction $[8,9]$.

\section{Material and Methods}

\section{Animals}

Animals were housed individually and provided with laboratory chow and water ab libitum. All experiments were approved by the Beth Israel Deaconess Medical Center Animal Care and Use Committee and the Harvard Medical Area Standing Committee on Animals and conformed to the US National Institutes of Health guide-lines regulating the care and use of laboratory animals (NIH publication 5377-83, 1996).

\section{Surgical Preparation}

Yorkshire pigs of either sex ( 32 to $42 \mathrm{~kg}, \mathrm{n}=15$ ) were sedated with ketamine hydrochloride $(20 \mathrm{mg} / \mathrm{kg}$, intramuscularly; Abbott Laboratories, North Chicago, IL), and anesthetized with a bolus infusion of thiopental sodium, 5.0 to $7.0 \mathrm{mg} / \mathrm{kg}$ intravenously (Baxter Healthcare Corporation, Deerfield, IL), through an ear vein. Surgical preparation (sternotomy, tracheotomy, cannulation) were performed as previously described $[8,9]$. General anesthesia was induced (3\%) and maintained (1\%) with sevoflurane (Ultane; Abbott Laboratories). During cardiopulmonary bypass (CPB), propofol, 0.5 to $0.7 \mathrm{mg} / \mathrm{kg} / \mathrm{min}$ intravenously (Baxter Healthcare Corporation), was continuously infused through the jugular vein. Heparin sodium, 5,000 IU intravenously, and 1\% lidocaine, $5 \mathrm{~mL}$ intravenously (both Elkins-Sinn, Cherry Hill, NJ) were given before sternotomy. Heparin was administered at the same dose every 30 minutes to the end of the experiment.

Cardiopulmonary bypass and regional ischemia were performed as previously described [Fig 1; 8, 9]. Immediately after 30 minutes of regional ischemia, the ascending aorta was crossclamped and $4^{\circ} \mathrm{C}$ cardioplegia was administered antegrade through the aortic root using a cardioplegia needle (9F AR II aortic root cannula; Medtronics DLP, Grand Rapids, MI). The DSA hearts $(\mathrm{n}=6)$ received $4^{\circ} \mathrm{C}$ blood cardioplegia $(10 \mathrm{~mL} / \mathrm{kg})$. The DSA+DZX hearts $(n=6)$ received DSA cardioplegia containing diazoxide $(50 \mu \mathrm{M})$. Diazoxide was dissolved in dimethyl sulfoxide (DMSO; Fisher Scientific, Fair Lawn, NJ) before being added to DSA. The DMSO was added as vehicle sham to DSA cardioplegia (final concentration less than $0.1 \%)$. At 15 minutes of global ischemia, an additional $5 \mathrm{~mL} / \mathrm{kg}$ of DSA cardioplegia without diazoxide was readministered in both groups [8]. All hearts received topical hypothermia during global ischemia [8]. After 30 minutes of hypothermic global ischemia, the cross clamp and the snare from the LAD were released, the ice packing was removed and the hearts were kept on total CPB for 120 minutes of reperfusion. Control hearts $(n=3)$ received cardiopulmonary bypass and sham reperfusion only. 


\section{Tissue Samples}

Immediately after 120 minutes of reperfusion myocardial tissue samples (approximately 15 $\mathrm{mm} \times 30 \mathrm{~mm}$ each) from the area at risk (regional ischemic zone, 6 samples) and nonischemic area of left ventricle (global ischemic zone, 6 samples) consisting of epicardial, myocardial, and endocardial tissue were harvested separately, flash frozen, and then stored in liquid nitrogen before analysis.

\section{Western Blot Analysis}

Nuclear protein extracts were isolated from pooled heart tissue ( $\mathrm{n}=6$ for each group of hearts) as previously described [9-11]. Protein concentration was determined by BCA (bicinchoninic acid) protein assay reagent (Pierce, Rockford, IL). Nuclear protein $(20 \mu \mathrm{g})$ was separated by $6 \%$ SDS-polyacrylamide gel electrophoresis and transferred to nitrocellulose membranes as previously described [11]. Efficiency of protein transfer was confirmed by Ponceau S staining $[9,11]$. Blots were incubated with appropriate primary antibodies (1:200 dilution), washed and incubated with appropriate secondary antibodies (1:2000), and detected using ECL Plus detection system (Amersham Pharmacia Biotech, Piscataway, NJ), as previously described $[9,11]$.

Antibodies used in this study were STAT1, STAT3, phospho-STAT1 (Tyr701), phosphoSTAT3 (Tyr705), phospho-STAT3 (Ser727 [Cell Signaling Technology, Beverly, MA]), and phospho-STAT1 (Ser727 [Biosource International, Camarillo, CA]).

Semiquantitative analysis of Western blots was performed using an LKB Ultrascan XL laser densitometer (Pharmacia LKB, Piscataway, NJ). The integrals for each blot were calculated using the LKB GelScan XL software program for 1-D analysis (Pharmacia LKB).

\section{Electrophoretic Mobility Shift Assay}

The STAT1/3 DNA binding was determined using sis-inducible element (SIE) consensus oligonucleotides (Santa Cruz Biotechnology, Santa Cruz, CA) end-labeled with $\mathrm{T}_{4}$ polynucleotide kinase (New England BioLabs, Beverly, MA) and $\left[\gamma^{32} \mathrm{P}\right]$ ATP (Amersham Biosciences, Piscataway, NJ) [12]. The labeled SIE oligonucleotides were purified with ProbeQuant G-50 columns (Amersham Biosciences). Electrophoretic mobility shift assay (EMSA) was performed as previously described [12].

In brief, nuclear protein $(10 \mu \mathrm{g})$ was incubated for 30 minutes at room temperature in a reaction mixture containing the following: $20 \mathrm{mM}$ HEPES (4-(2-hydroxyethyl)-1piperazineethanesulfonic acid, $\mathrm{pH} 7.9), 50 \mathrm{mM} \mathrm{KCl}, 5 \mathrm{mM} \mathrm{MgCl} 2,1 \mathrm{mM}$ EDTA (ethylenediaminetetraacetic acid, pH 8.0), $1 \mathrm{mM}$ DTT (dithiothreitol), $12.5 \%$ glycerol, $1 \mu \mathrm{g}$ poly(deoxy-inosine, deoxy-cytosine [Santa Cruz Biotechnology]), and 100,000 cpm labeled SIE oligonucleotides [12,13]. For competition experiments, a 100-fold molar excess of unlabeled (cold) SIE oligonucleotides was added to the reaction mixture. Supershift experiments were performed as above but with the addition of $2 \mu \mathrm{g}$ anti-STAT3 (Cell Signaling Technology) to the reaction mixture. The DNA(SIE)-protein complexes were separated by $4 \%$ native poly-acrylamide gel electrophoresis for 2 hours at $35 \mathrm{~mA}$.

To resolve STAT1/3 hetero-, homo-dimers, a separate set of gels was run for 6 hours at 35 $\mathrm{mA}$, as described by Zhong and coworkers [13]. Autoradiography was performed for 12 hours at $-80^{\circ} \mathrm{C}$ using intensifying screens.

\section{Statistical Analysis}

The mean \pm SEM is shown for all variables. Statistical significance was determined by a mixed models analysis of variance (MM-ANOVA), which allows for both fixed and random 
effects and allows for more than one observation per animal (regional and global). In the analysis, when more than one observation was used from an animal both the site of ischemia (regional or global) was treated as a repeated measurement and the specific animal was incorporated as a random effect. All analyses were done in SAS for Windows, release 8.02 (SAS Institute, Cary, NC). Post hoc comparisons were made using a Bonferroni correction.

\section{Results \\ Effects of DSA and DSA+DZX Cardioplegia on Total STAT1/3}

Total STAT1 and STAT3 levels were significantly decreased in both regional and global ischemic zones as compared with control ( $p<0.0001$ for each; Fig 2$)$. There was no significant difference in STAT 1 or STAT3 levels between regional and global ischemic zones ( $p=0.6525$ and $p=0.7427$, respectively).

\section{Effects of DSA and DSA+DZX Cardioplegia on Serine Phosphorylation of STAT1/3}

Serine phosphorylation of nuclear STAT1 and STAT3 (pSer-STAT1, pSer-STAT3) was evident in Control hearts. There was no significant difference in pSer-STAT3 levels within or between control, DSA, and DSA+DZX hearts ( $p=0.7073$; Figs 2 and 3 ).

The pSer-STAT1 levels were significantly decreased in both regional and global ischemic zones in both DSA and DSA+DZX hearts as compared with control $(p<0.0001$; Figs 2 and $3)$. There was no significant difference in pSer-STAT 1 levels between DSA and DSA+DZX hearts in the global and regional ischemic zones ( $p=0.9825$; Figs 2 and 3$)$.

\section{Effects of DSA and DSA+DZX Cardioplegia on Tyrosine Phosphorylation of STAT1/3}

There was no detectable tyrosine phosphorylation of proapoptotic STAT1 (pTyr-STAT1) or antiapoptotic STAT3 (pTyr-STAT3) in Control hearts receiving cardiopulmonary bypass and sham reperfusion (Figs 2 and 3). However, in DSA and DSA+DZX hearts that received cardiopulmonary bypass and ischemia and reperfusion, pTyr-STAT1 and pTyr-STAT3 were detectable in both the regional and global ischemic zones (Figs 2 and 3).

Proapoptotic pTyr-STAT1 levels were significantly increased in regional as compared with global ischemic zones in both DSA and DSA+DZX hearts ( $p=0.0047$; Figs 2 and 3).There was no significant difference in proapoptotic pTyr-STAT1 between DSA and DSA+DZX hearts within the regional or within the global ischemic zones (Fig 3).

Antiapoptotic pTyr-STAT3 levels were significantly increased in the global ischemic zones in both DSA and DSA+DZX hearts and in the regional ischemic zone in DSA+DZX hearts (Figs 2 and 3). No significant difference in antiapoptotic pTyr-STAT3 levels were observed between DSA and DSA+DZX in the global ischemic zone $(p=0.1161)$ and DSA+DZX in the regional ischemic zone ( $p=0.0516$; Figs 2 and 3$)$. However, in the regional ischemic zone, antiapoptotic pTyr-STAT3 levels were significantly decreased in DSA as compared with DSA+DZX $(p<0.0001)$ and were significantly decreased as compared with DSA and DSA+DZX in the global ischemic zones ( $p<0.0001$; Figs 2 and 3$)$.

\section{Effects of DSA and DSA+DZX Cardioplegia on DNA Binding of STAT1/3}

There was no detectable STAT1 or STAT3 DNA-binding in control hearts (Fig 4A, lane 2, 4B, lane 2). Control samples showed no detectable DNA binding bands for STAT1/STAT1, STAT1/STAT3, or STAT3/STAT3 (Fig 4A, lane 2, 4B, lane 2).

Binding bands for STAT1/STAT1, STAT1/STAT3, and STAT3/STAT3 DNA were detected for both DSA and DSA+DZX hearts in both regional and global ischemia zones (Fig 4B, 
lanes 3 to 6). There was decreased signal intensity for the STAT3/STAT3 DNA binding in the regional ischemic zone of DSA hearts (Fig 4B, lane 3).

The specificity of STAT1/STAT1, STAT1/STAT3, and STAT3/STAT3 DNA binding was confirmed by antibody supershift assay. The migration of STAT3/STAT3 and STAT1/ STAT3 DNA binding bands in both regional and global ischemia zones was retarded by the addition of anti-STAT3 antibody (Fig 4B, lanes 7, 9). There was no retardation of the STAT1/STAT1 DNA binding band with the addition of anti-STAT3 antibody (Fig 4B, lanes 7, 9).

Confirmation of the DNA binding of STAT1/STAT1, STAT1/STAT3, and STAT3/STAT3 was obtained through the addition of a 100-fold molar excess of unlabeled (cold) SIE oligonucleotides to compete-out binding to labeled SIE. No detectable DNA binding bands were observed for either DSA or DSA+DZX in either the regional or global ischemia zones in the presence of 100-fold molar excess cold SIE (Fig 4A, lane 7, 4B, lanes 8, 10).

To confirm that the significantly increased antiapoptotic pTyr-STAT3 levels with DSA + DZX cardioplegia in the regional ischemic zone were associated with increased DNA binding, a competitive EMSA using admixtures of DSA and DSA+DZX nuclear proteins was performed (Fig 5A, B). Our results show there was a significant increase in STAT3/ STAT3 DNA binding in DSA+DZX (Fig 5, lane 4) as compared with DSA ( $p=0.0090$; Fig 5 , lane 1). The admixtures of DSA+DZX (5 and $7.5 \mu \mathrm{g}$ nuclear protein, respectively) to DSA (5 and $2.5 \mu \mathrm{g}$ nuclear protein, respectively) significantly increased STAT1/STAT3 and STAT3/STAT3 DNA binding (Fig 5 lanes 2, 3; Fig 5B). There was no significant difference in STAT1/STAT1 DNA binding between DSA and DSA+DZX hearts at any admixture (Fig 5A, lanes 1 to 4; Fig 5B).

\section{Comment}

Previously, we have shown that DSA+DZX cardioplegia significantly decreases mitochondrial regulated (intrinsic) apoptosis as compared with DSA cardioplegia alone [9]. The mechanism for this enhanced cardioprotection was unknown but we believed, based on earlier work, that alterations in signal transducers and activators of transcription may play a role $[9,14]$. To further investigate this hypothesis, we have examined the effects of DSA and DSA+DZX cardioplegia on STAT1/3 serine and tyrosine phosphorylation and DNA binding.

Previous studies have shown that tyrosine phosphorylation of STATs is essential for dimerization and translocation into the nucleus to allow for DNA binding [6, 7]. Current models suggest that when there is no tyrosine phosphorylation, as in the case of our control groups, no nuclear translocation or DNA binding is observed. However; it has been shown that unphosphorylated STAT1 and STAT3 can bind to DNA transcription factors and modulate selective constitutive gene expression in oncogenesis $[15,16]$. The mechanism for this regulation is novel and is due either to the direct effect of STAT binding to specific promoters or through the associative indirect activation of unrelated genes [16]. It has also been suggested that the STAT's could be activated by mechanisms other than phosphorylation; however, further research is required to establish this as a regulatory mechanism.

In our model, there is no detectable tyrosine phosphorylation of either STAT1 or STAT3 in control hearts (no ischemia; Figs 2, 3), and there is no detectable STAT1 or STAT3 DNA binding bands (Fig 4). It should be noted that this is an experimental model and specific binding to the SIE element only was assayed. 
The role of serine phosphorylation of STAT1/3 is unknown. There is currently no evidence to indicate that serine phosphorylation has any effect on the nuclear translocation of STAT1/3, or on nuclear DNA binding. However, some data are available to show that increased serine phosphorylation of STAT1 is associate with increased interferon-gamma transcription factor activity [17]. It has been proposed that serine phosphorylation of STAT1/3 acts in coordination with tyrosine phosphorylation to allow signal transduction, either by direct DNA binding or possibly through an unidentified cofactor [17].

Our results show that there is no significant difference in serine phosphorylation of STAT3 within or between any group (Figs 2 and 3). Our results also show that while there is a significant decrease in serine phosphorylation of STAT1 with global and regional ischemia there are no significant differences within or between regional and global ischemia groups (Figs 2 and 3). These data do not provide any mechanistic link for serine phosphorylation for either STAT1 or STAT3 in the modulation of apoptosis.

Our studies do show changes in tyrosine phosphorylation of STAT1/3 with DSA and DSA +DZX cardioplegia STAT1 and STAT3 tyrosine phosphorylation has been previously shown to be associated with the modulation of apoptosis. Increased STAT1 tyrosine phosphorylation and DNA binding has been associated with proapoptotic responses. Previous studies have shown that STAT1-deficient mice develop spontaneous and chemically induced tumors more rapidly than wild-type mice and that STAT-1-deficient cells are more resistant to agents that induce apoptosis $[2,18]$. It has also been shown that STAT1 modulates cardiac myocyte apoptosis after simulated ischemia and reperfusion [3, 4]. In contrast, depletion of STAT-3 has been shown to abolish the cardioprotection afforded by preconditioning [19].

Our data show that STAT1 tyrosine phosphorylation is significantly increased in the regional ischemic zone as compared with the global ischemic zone in both DSA and DSA +DZX hearts (Figs 2, 3). That would imply that the levels of apoptosis should increase in the regional as compared with the global ischemic zone and that this increase would be similar in both DSA and DSA+DZX cardioplegia hearts. However, our earlier studies have shown that DSA+DZX significantly decreases apoptosis in the regional ischemic zone as compared with DSA [9].The mechanism for this can be explained by the differential response in antiapoptotic STAT3 tyrosine phosphorylation and DNA binding to DSA+DZX cardioplegia.

Our results show that tyrosine phosphorylation of STAT3 is significantly increased in the regional ischemic zone with DSA+DZX and is associated with significantly decreased apoptosis as compared with DSA [9]. These data suggest that apoptosis may be the result of a shifting of the balance between proapoptotic and antiapoptotic levels.

We speculate that ischemia induces an increase in proapoptotic STAT1 levels that increases apoptosis. With DSA cardioplegia antiapoptotic STAT3 levels are significantly decreased in the regional as compared with the global ischemic zone. This differential leads to a shift in balance towards the induction of apoptosis by proapoptotic STAT1. In DSA+DZX cardioplegia, however, there is a significant increase in antiapoptotic STAT3 levels as compared with DSA such that no difference between regional and global ischemic zones is observed. The increased antiapoptotic STAT3 levels shift the balance from proapoptosis as seen in DSA hearts to antiapoptosis in DSA+DZX hearts and decrease apoptosis.

The mechanism through which the opening of mitoK $_{\mathrm{ATP}}$ channels with diazoxide significantly increases antiapoptotic pTyr-STAT3 levels and DNA binding remains to be fully elucidated. In previous reports we have speculated that the preservation of mitochondrial structure and function by DSA+DZX cardioplegia plays an important role in 
the modulation of ischemia/reperfusion injury $[8,9]$. Our data have also demonstrated that apoptosis occurs via the mitochondrial regulated intrinsic apoptosis pathway and involves changes in the bax/bcl 2 ratio $[8,9]$. It has been shown that STAT3 activates bcl 2 expression [20]. This activation would be expected to alter the ratio of proapoptotic bax to antiapoptotic bcl2 and would be expected to decrease apoptosis, as we have shown in our model.

It is possible that cofactors may be involved. Previous reports have shown that c-Jun interacts with STAT3 and enhances STAT3 DNA-binding [19-23]. In this report, we attempted to examine whether c-Jun interacts with STAT3 in our model by coimmunoprecipitation with anti-STAT3 antibodies followed by Western blot analysis with anti-c-Jun antibodies. However, we failed to detect c-Jun in the STAT3 coprecipitates (data not shown), indicating that if alternative cofactors are involved further research and better antibodies will be needed for identification.

In this paper, we have extended our earlier observations using the clinically relevant in situ blood perfused heart model of acute myocardial infarction $[8,9]$. This model allows for the investigation of regional ischemic injury similar to that observed in the patient under conditions which more closely approximate those experienced during surgery. In this model, a region of the leftventricle is made ischemic by ligation of a portion of the left anterior descending artery. Cardioplegic arrest and cross-clamping occurs resulting in global ischemia and then the snare and the crossclamp are released after bypass and the heart is reperfused for 120 minutes.

Our model is a large-animal model and is clinically relevant; however, the usual caveats regarding direct relevance to humans must be considered. We readminister cardioplegia every 15 minutes, as this is our standard clinical practice. The length of ischemia was based on our previous studies showing it allows for effective comparative analysis [8, 9].

Diazoxide was included only in the first administration of cardioplegia as diazoxide has a long half life and binds to hemoglobin, and added administration of cardioplegia containing diazoxide was found to significantly decrease mean arterial pressure $[8,9]$.

The role of hypothermia in the prevention of apoptosis has been previously reviewed, and it has been shown that mild hypothermia is somewhat protective while deep hypothermia increases apoptosis [24]. In our model we administer cold $4^{\circ} \mathrm{C}$ cardioplegia. No attempt at modulation of temperature beyond these limits has been performed. We speculate that deep hypothermia may decrease transcription and thus decrease the antiapoptotic effects of STAT modulation; however, further studies are needed to support this hypothesis.

In conclusion, our data indicate that STAT1 tyrosine phosphorylation and DNA binding are associated with increased apoptosis. The addition of diazoxide to DSA cardioplegia modulates apoptosis by significantly increasing tyrosine phosphorylation of STAT3 and its DNA binding, and represents an additional modality for enhancing myocardial protection. Our work also raises an interest that the STAT3 signaling pathway can be targeted to stimulate protective process attenuating ischemia/reperfusion injury.

\section{References}

1. Bolli R, Dawn B, Xuan YT. Role of the JAK-STAT pathway in protection against myocardial ischemia-reperfusion injury. Trends Cardiovasc Med. 2003; 13:72-9. [PubMed: 12586443]

2. Stephanou A, Latchman DS. STAT-1: a novel regulator of apoptosis. Int J Exp Pathol. 2003; 84:239-4. [PubMed: 14748743]

3. Stephanou A. Activated STAT-1 pathway in the myocardium as a novel therapeutic target in ischaemia/reperfusion injury. Eur Cytokine New. 2002; 13:401-3. 
4. Stephanou A, Scarabelli TM, Townsend PA, et al. The carboxyl-terminal activation domain of the STAT-1 transcription factor enhances ischemia/reperfusion-induced apoptosis in cardiac myocytes. FASEB J. 2002; 16:1841-3. [PubMed: 12223448]

5. Mascareno E, El-Shafei M, Maulik N, et al. JAK/STAT signaling is associated with cardiac dysfunction during ischemia and reperfusion. Circulation. 2001; 104:325-9. [PubMed: 11457752]

6. Levy DE, Darnell JE Jr. Stats: transcriptional control and biological impact. Nat Rev Mol Cell Biol. 2002; 3:651-62. [PubMed: 12209125]

7. Leonard WJ, O'Shea JJ. JAKS and STATS: biological implications. Annu Rev Immunol. 1998; 16:293-322. [PubMed: 9597132]

8. Wakiyama H, Cowan DB, Toyoda Y, Federman M, Levitsky S, McCully JD. Selective opening of mitochondrial ATP-sensitive potassium channels during surgically induced myocardial ischemia decreases necrosis and apoptosis. Eur J Cardiothorac Surg. 2002; 21:424-33. [PubMed: 11888758]

9. McCully JD, Wakiyama H, Cowan DB, Federman M, Levitsky S. Diazoxide amelioration of myocardial injury and mitochondrial damage during cardiac surgery. Ann Thorac Surg. 2002; 74:2138-46. [PubMed: 12643408]

10. Andrews NC, Faller DV. A rapid micropreparation technique for extraction of DNA-binding proteins from limiting numbers of mammalian cells. Nucleic Acids Res. 1991; 19:2499. [PubMed: 2041787]

11. McCully JD, Wakiyama H, Hsieh Y-J, Jones M, Levitsky S. Differential contribution of necrosis and apoptosis in myocardial ischemia/reperfusion injury. Am J Physiol Heart Circ Physiol. 2004; 286:H1923-35. [PubMed: 14715509]

12. Garner MM, Revzin A. A gel electrophoresis method for quantifying the binding of proteins to specific DNA regions: application to components of the Escherichia coli lactose operon regulatory system. Nucl Acids Res. 1981; 9:3047-60. [PubMed: 6269071]

13. Zhong Z, Wen Z, Darnell JE Jr. STAT3: a STAT family member activated by tyrosine phosphorylation in response to epidermal growth factor and interleukin-6. Science. 1994; 264:958. [PubMed: 8140422]

14. Matsuda H, McCully JD, Levitsky S. Inhibition of RNA transcription modulates magnesium supplemented potassium cardioplegia protection. Ann Thorac Surg. 2000; 70:2107-12. [PubMed: 11156129]

15. Chatterjee-Kishore M, Wright KL, Ting JP, Stark GR. How Stat1 mediates constitutive gene expression: a complex of unphosphorylated Stat1 and IRF1 supports transcription of the LMP2 gene. EMBO J. 2000; 19:4111-22. [PubMed: 10921891]

16. Yang J, Chatterjee-Kishore M, Staugaitis SM, et al. Novel roles of unphosphorylated STAT3 in oncogenesis and transcriptional regulation. Cancer Res. 2005; 65:939-47. [PubMed: 15705894]

17. Decker T, Kovarik P. Serine phosphorylation of STATs. Oncogene. 2000; 19:2628-37. [PubMed: 10851062]

18. Meraz MA, White JM, Sheehan KCF, et al. Targeted disruption of the Stat 1 gene in mice reveals unexpected physiologic specificity in the JAK-STAT signaling pathway. Cell. 1996; 84:431-42. [PubMed: 8608597]

19. Smith RM, Suleman N, Lacerda L, et al. Genetic depletion of cardiac myocyte STAT-3 abolishes classical preconditioning. Cardiovasc Res. 2004; 63:611-6. [PubMed: 15306216]

20. Hattori R, Maulik N, Otani H, et al. Role of STAT3 in ischemic preconditioning. J Mol Cell Cardiol. 2001; 33:1929-36. [PubMed: 11708838]

21. Yoo YJ, Wang W, Desiderio S, Nathans D. Synergistic activity of STAT3 and c-Jun at a specific array of DNA elements in the alpha 2-macroglobulin promoter. J Biol Chem. 2001; 276:26421-9. [PubMed: 11319221]

22. Zhang X, Wrzeszczynska MH, Horvath CM, Darnell JE Jr. Interacting regions in Stat3 and c-Jun that participate in cooperative transcriptional activation. Mol Cell Biol. 1999; 19:7138-46. [PubMed: 10490649]

23. Gross ER, Hsu AK, Gross GJ. The JAK/STAT pathway is essential for opioid-induced cardioprotection: JAK2 as a mediator of STAT3, Akt, and GSK-3b. Am J Physiol Heart Circ Physiol. 2006; 291:H827-34. [PubMed: 16517948] 
24. Valen G. The biology of apoptosis and its implications for cardiac function and viability. Ann Thorac Surg. 2003; 75:656-60. Suppl. 


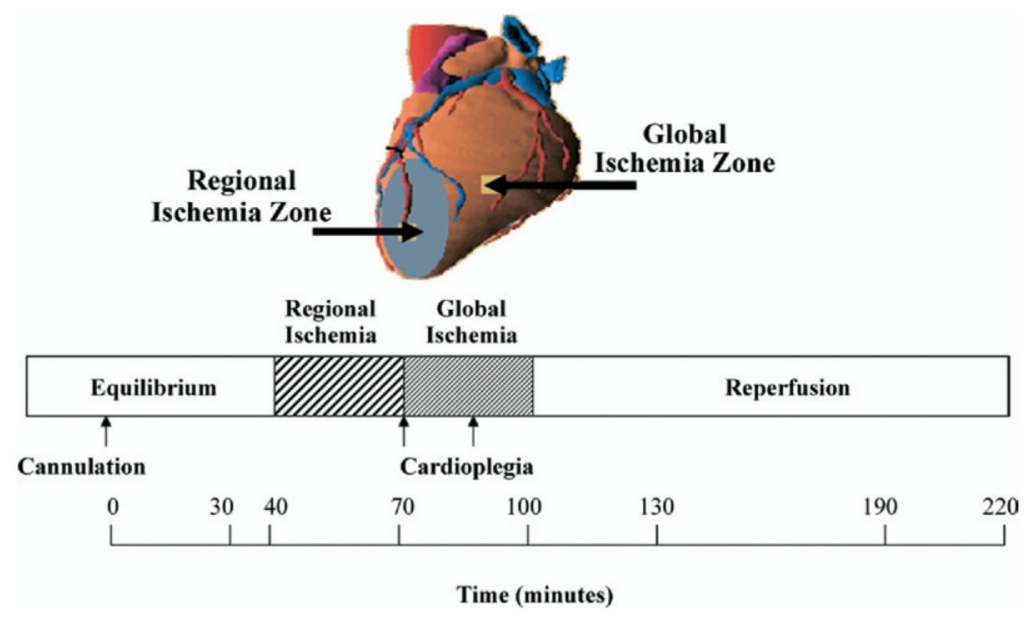

Fig 1.

Experimental schema of acute myocardial infarct model in the in situ pig heart. Pigs were cannulated placed on cardiopulmonary bypass. After 30 minutes of stabilization, hearts were subjected to regional ischemia by snaring of the distal portion of the left anterior descending artery (LAD). Immediately after 30 minutes of normothermic regional ischemia, the aorta was cross-clamped, and cold blood magnesium supplemented potassium (DSA) cardioplegia or DSA with the addition of $50 \mu \mathrm{Mol} / \mathrm{L}$ diazoxide (DSA+DZX) was administered through the aortic root. The hearts were then subjected to hypothermic global ischemia. After 30 minutes of hypothermic global ischemia, the cross clamp and the snare were released and the hearts reperfused for 120 minutes. Tissue samples from the regional ischemia and global ischemia zones were obtained and quick frozen in liquid nitrogen before isolation of nuclear proteins. 


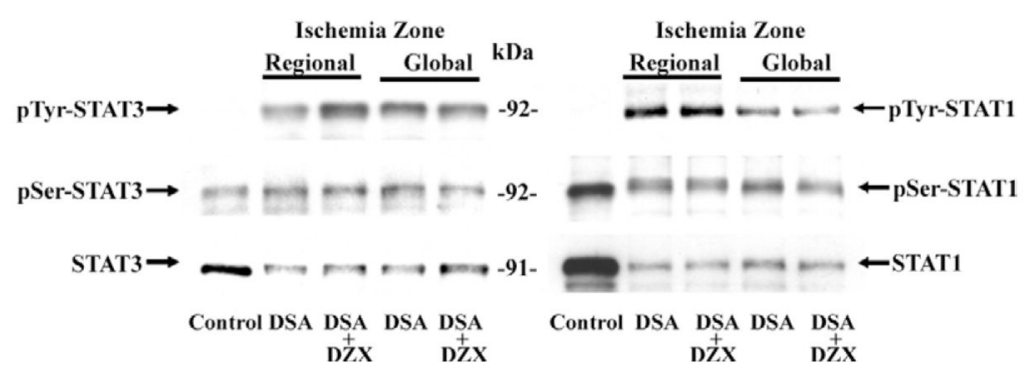

Fig 2.

Representative Western blots of nuclear protein signal transducers and activators of transcription (STATs) STAT1, STAT3, and tyrosine (pTyr)and serine (pSer) phosphorylation levels in control, magnesium supplemented potassium (DSA) and DSA plus diazoxide (DSA+DZX) hearts. Control hearts were subjected to sham regional and global ischemia. Regional ischemia and global ischemia zones are indicated. Molecular mass is shown for each gel. 

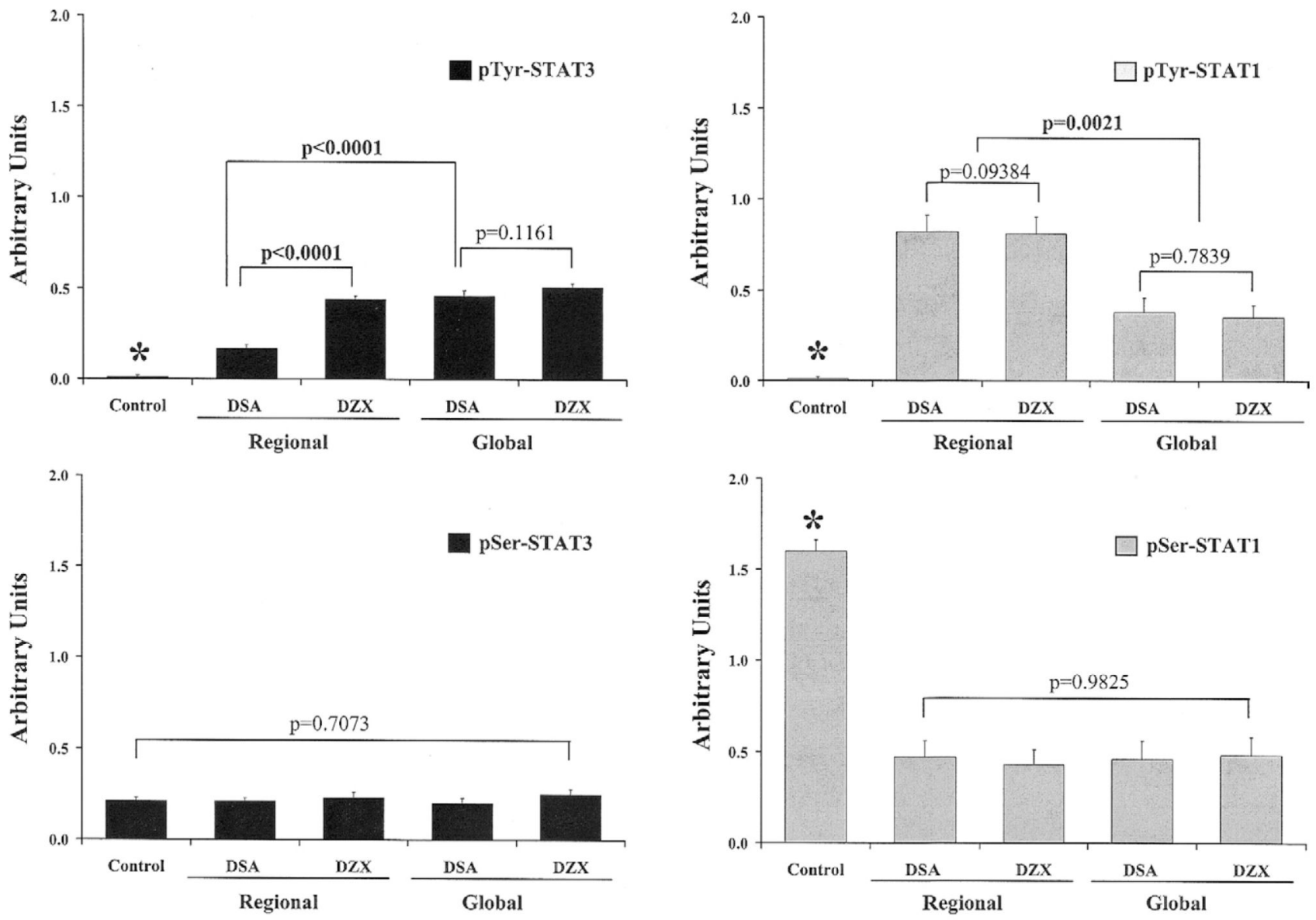

Fig 3.

Semiquantitative analysis of Western blot signal transducers and activators of transcription (STATs) STAT1, STAT3, tyrosine (pTyr), and serine (pSer) phosphorylation levels. All results are shown as the mean \pm SEM for $n=6$ separate blots. Densitometry is shown in arbitrary units. Significant differences between groups are shown in bold type. Significant difference at $\mathrm{p}<0.0001$ versus regional and global ischemic zones is indicated by an asterisk $(*)$. 
$\mathbf{A}$

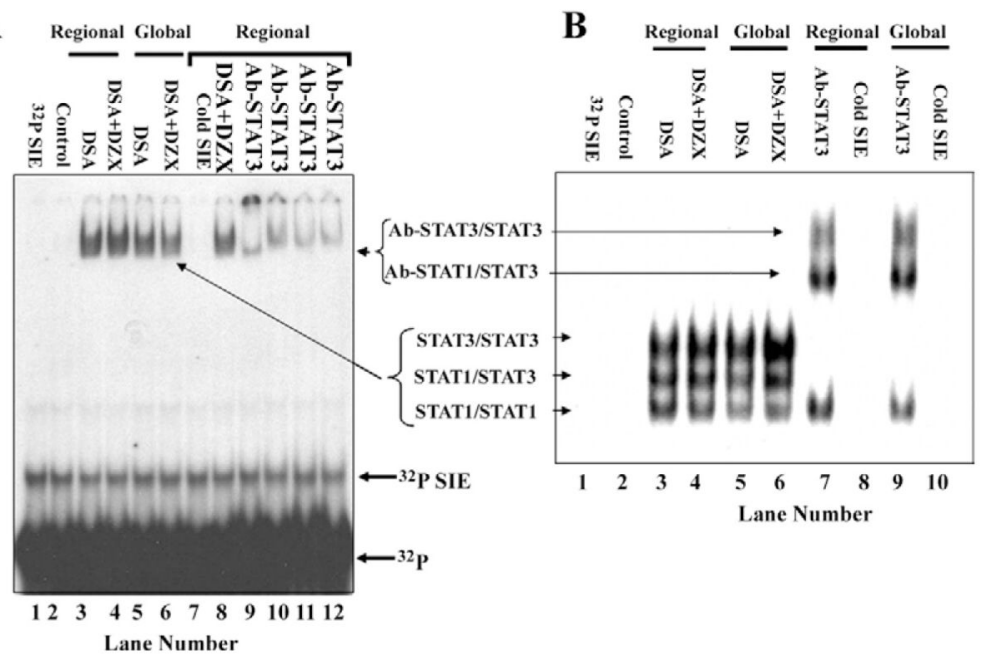

Fig 4.

Representative electrophoretic mobility shift assay (EMSA) of signal transducers and activators of transcription (STATs) STAT1, STAT3 DNA binding. (A) Representative EMSA run for 2 hours at $35 \mathrm{~mA}$. Supershift bands are shown in lanes 9 to 12. (B)

Representative EMSA run for 6 hours at $35 \mathrm{~mA}$, as described by Zhong and coworkers [13], to allow for identification of STAT1/STAT1, STAT1/STAT3, and STAT3/STAT3 dimer DNA binding. The STAT1, STAT3 DNA binding in DSA and DSA+DZX in regional and global ischemia zones is shown. Control hearts were subjected to sham regional and global ischemia. The ${ }^{32} \mathrm{P}$, unincorporated $\left[\gamma-{ }^{32} \mathrm{P}\right]$ ATP, ${ }^{32} \mathrm{P}$ SIE, $\left[\gamma^{-32} \mathrm{P}\right]$ labeled sis-inducible element consensus oligonucleotides; cold SIE, unlabeled SIE (100-fold molar excess); AbSTAT3, supershift using $2 \mu \mathrm{g}$ anti-STAT3 antibody. STAT1/STAT1, STAT1/STAT3, STAT3/STAT3, and supershift STAT1/STAT3 (Ab-STAT1/STAT3) and STAT3/ STAT3 (Ab-STAT3/ STAT3) are indicated. 
A

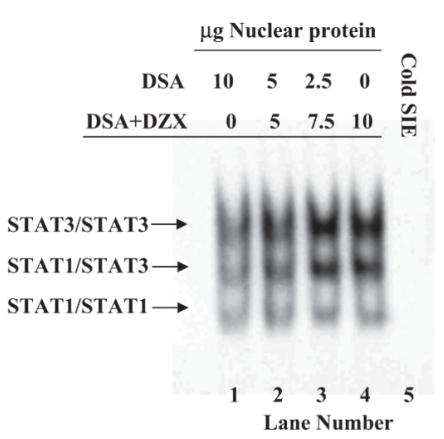

B

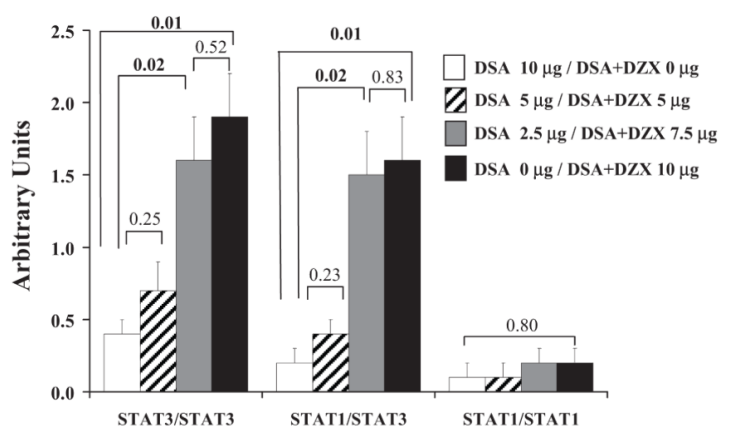

Fig 5.

(A) Representative competitive electrophoretic mobility shift assay (EMSA) using admixtures of magnesium supplemented potassium (DSA) and DSA containing diazoxide (DSA+DZX) nuclear proteins. Nuclear protein content for each EMSA is shown. Signal transducers and activators of transcription (STATs) STAT1/STAT1, STAT1/STAT3, and STAT3/STAT3 dimer DNA binding for DSA, DSA+DZX (lanes 1 and 4), and DSA and DSA+DZX admixtures (lanes 2 and 3) are shown. Cold sis-inducible element (SIE), unlabeled SIE (100-fold molar excess) is shown in lane 5. (B) Semiquantitative analysis of STAT3/STAT3, STAT1/STAT3, and STAT1/STAT1 dimer DNA binding. The DSA and DSA+DZX admixtures are indicated (open bars = DSA $10 \mu \mathrm{g} / \mathrm{DSA}+\mathrm{DZX} 0 \mu \mathrm{g}$; hatched bars = DSA $5 \mu \mathrm{g} / \mathrm{DSA}+\mathrm{DZX} 5 \mu \mathrm{g}$; gray bars = DSA $2.5 \mu \mathrm{g} / \mathrm{DSA}+\mathrm{DZX} 7.5 \mu \mathrm{g}$; black bars $=$ DSA $0 \mu \mathrm{g} / \mathrm{DSA}+\mathrm{DZX} 10 \mu \mathrm{g}$ ). All results are shown as the mean \pm SEM for $\mathrm{n}=4$ separate blots. Densitometry is shown in arbitrary units. Significant differences between groups are shown in bold type. 